\title{
Proposition for the Innovation of the Rehabilitation Medicine -Retrospection and Reflection Related to Mechanism of Proprioceptive Neuromuscular Facilitation-
}

\author{
Kunihiko Fukui, MD. PhD ${ }^{1)}$, Tetsuhiko Kimura, MD. PhD ${ }^{2)}$, TAKIZAWA Shigeo, MA ${ }^{\text {3) }}$ \\ ${ }^{1)}$ Honorary chairman of Biophilia Rehabilitation Academy \\ ${ }^{2)}$ President of Biophilia Rehabilitation Academy \\ ${ }^{3)}$ CEO of International Division of Biophilia Rehabilitation Academy \\ Biophilia Rehabilitation Academy \\ 252-0804 4-24-5, Shonandai, Fujisawa-shi, Japan
}

\begin{abstract}
The priority of Proprioceptive Neuromuscular Facilitation (PNF) to hemiplegic patients on the affected side must be reconsidered due to a lack of effect in its procedure. By the improvement of the theory and techniques of $\mathrm{PNF}$, the improvement of therapeutic effects on hemiplegia patients could be expected in connection with their progress, however the outcome of medical examination has hardly been changed on hemiplegia patients at the clinical aspect over the last 40 years. Although PNF is thought to be more suitable for the normalization of a movement pattern to perform synthetic coordinated movements by affected side priority, it takes too long to lead to the active contraction of the peripheral muscles. Sometimes, even the atrophy of an unaffected side and the trunk can be seen. In considering the PNF of unaffected side priority the effect of facilitation is larger than that of affected side priority. Therefore, if the unaffected priority is used a smaller number of patients will be bedridden by atrophy and contracture. From the recent research on new born power of a neural stem cell (NSC), the elucidation of the mechanism for the effectiveness of the kinetic rehabilitation by the unaffected side priority enables us to expect the functional recovery of a damaged area. The NSC would also affect to the rehabilitation in the realm of the nervous system. It's recommended to try to use and get the evidence of PNF by unaffected side priority.
\end{abstract}

KEY WORDS; Facilitation, Unaffected side initiative, Neural stem cell, Unaffected side new center, Spasticity control

\section{Verification of the Proprioceptive Neuromuscular Facilitation}

\subsection{The neuromuscular facilitation techniques}

Mainly, Rood, Bobath, Brrunstrome and PNF methods are known as neuromuscular facilitation techniques. These have been around for approximately 60 years since their arrival in Japan in the late 1940s. They make overflow motion start from a strong muscle toward weaker muscles. Then, they cause cooperation of muscles, and those muscles contract as synergist muscles, increasing the effect of movement. That is the overflow that happens to the synergist muscles and the antagonist muscles as a result of facilitation, control, relaxation etc.

It is expected that the whole muscle will be recovering by and by if even one muscle among all, which has a comparatively light disability becomes a leader and begins to move, even if there is paralysis. In affected side muscles, a muscle which has a comparatively lower level of paralysis is made to irradiate to the one with higher level of paralysis and causes contraction. Since there are several techniques other than PNF, the physical therapists perform strengthening and control of muscles according to combinations of neuromuscular facilitation techniques they chose. This should bring some effect, but if the atrophy and/or contracture of the paralysis muscle and a joint happen and the patient shows a disuse syndrome, the purpose cannot be attained even if the irradiation is applied. If the disabled level of the leader muscle is higher and the paralysis is more serious, the effect is difficult to see.

\subsection{The effect of the neuromuscular facilitation techniques}

When comparing the results of the typical technique of neuromuscular facilitation technique (FT) and the traditional technique (TT) (muscular power reinforcement, range of motion (ROM) ex. and Motion Training), there are very few effective results deriving from them.

The investigation of 36 pieces articles on rehabilitation has been done by the joint guideline committee 1). The committee consists of 5 academies, the Japanese Society of Neurology, the Japanese Stroke Society, 
the Japan Neurosurgical Society, the Japanese Society of Neurological Therapeutics and The Japanese Association of Rehabilitation Medicine and 3 research groups of the Ministry of Health, Labor and Welfare, the cerebral infarction, intracerebral bleeding, and subarachnoid bleeding. The investigation was based on the evidence they collected over 30 years, from 1960-1990.

In respect to activities of daily living (ADL) and walking ability are made a standard for comparison; the following points are raised in these papers that described the differences of the effects of FT and TT.

(i) The Bobath method and the PNF method didn't show a difference from the TT method. (Dickstein et al) ${ }^{2)}$

(ii) The group which had done the Rood and Bobath methods didn't show any difference from the group which had done the TT method. (Logigian et al). ${ }^{3)}$

(iii) No difference was seen when PNF and the Brunnstrome method were added to the TT group. (Stern et al) ${ }^{4)}$

(iv) A significant difference in the improvements of walking and hand function didn't show even if Neurodevelopmental exercise (Davis) was performed in rotation by five-week shift. (Wagenaar et al) ${ }^{5 \text { ) }}$

As a result, there's no substantiation to be considered in the improvement of the quality of movement by FT.

\subsection{The examples which PNF didn't show any effectiveness on patients of hemiplegia}

In past medical treatment, when muscular power is generally in a low level of 1 or 2 after the onset, the muscle cannot be used at all because the atrophy and deformation appears before the PNF effect shows up. When 2 or more differences exist in the muscular power of an agonist muscle and the antagonist, the weaker muscle cannot counterwork the effects of the atrophy and deformations. It is because of the proprioceptive imperfections that PNF doesn't show much effect when disturbance of sensation is strong on the whole. Though it is not clear why there is a perceptual disturbance, it is possible that a major cognitive disturbance is a main cause when aphasia, apraxia, and agnosia are highly advanced.

Flection contracture is examined as an example. The contractile force of agonist needs to exceed the tension at the time of relaxation of an antagonist in order to reach even an active movement state. We consider that it has a relation with the appearance of spastic paralysis. A concept is shown in FIG. 1 by considering the following three points as a cause:

(i) No active movement is seen because the muscle of heavy paralysis cannot resist a powerful antagonist.

(ii) If an antagonist is powerful and strain power is strong, the atrophy and deformation happen before the PNF effect shows up.

(iii) Agonist muscles cause atrophy, rigidity, degeneration, deformity, displacement, etc. and will end up in disuse.

A joint will also deform due to the unbalance of muscular power, and a resulting in atrophy, displacement, deformation, etc. under the influence of disuse and this extremity will end up in disuse. These phenomena will occur within several months.

\section{Neural Stem Cell and plasticity of an adult central nervous system}

Activity of a neural stem cell, the activity of the cell producing from neurotransmitter and a neurotrophic factor are considered. It has been evidenced that it is useful for functional recovery of a damaged area with these substances being secreted from the summoned neural stem cell.

\subsection{Neural stem cell}

The formation of neural circuits and the increase of the number of synapses by new birth have great influence on the growth of a young person. It is been clarified that the neural stem cell (NSC) is equipped with the capability to specialize in nerve cell and nerve committed stem cell, with both existing in the brain of a matured rat, thereby explaining that the potential function to form a new circuit resides with a newly produced nerve cell and is due to the strengthening of remaining synapse function or the rebirth of an embryonic stem cell or a neural stem cell which gather after their maturity. ${ }^{6}$

Van Praag $\mathrm{H}$ and others ${ }^{7), 8)}$ assumed that in the hippocampus of a rat, several thousand nerve cells are newly generated each day. In addition, they are reporting that performing suitable activity and living in diversified environments eminently promote the rebirth of a nerve cell compared with the case of no activities (exercises). The rebirth of a nerve cell is considered to continue along the whole life of a human being as it does in a rat. Evidence is likely to show that a muscle is required to perform at least active or 
resistant movements in order to reproduce or increase NSC and plasticity of an adult central nervous system.

\subsection{Possibility for recovery of functional damage}

It was clearly shown in the new research by Jaime Imitola and others ${ }^{9), 10), 11)}$ that a substance called chemokine was secreted from the neuronal tissues that were damaged or inflamed, and along with this, NSC was responsive. They have come to a conclusion that NSC moved toward an infarct domain in vivo (including the movement from the opposite side of the hemisphere). This result was obtained by a clarification of the movement of NSC from the region of transplantation which is away from a pathological change. NSC then twist together with chemokine producing cell when the doctors guide the nerve damage with a mouse and incubate cells in a fluorescent stain enabling them to pursue the trajectory of cells under a microscope, investigating whether SDF-1A (Stromal Cell-Derived Factor 1 Alpha) guides NSC in vivo or not. It has been reported locally that once cells arrive at a damaged area, NSC may be included in the niche ${ }^{*}$ of the cell that has the restoration signal expressed on a temporary basis. Although it was already known that NSC will move in accordance with an atypical course through tissue substance, they have predicted a possibility of NSC carrying out long-distance movement to the damaged area of a central nervous system with correct directionality.

\subsection{The elucidation of a mysterious result}

As previously stated the rebirth or increase of a nerve cell is considered to continue along the whole life of a human being, a finding which became clear in research in recent years. Research is likely to show that a muscle is required to perform at least active or resistant movements in order for rebirth or increase of neural stem cells and plasticity of an adult central nervous system. Also, the report made by Jaime Imitola and others show the possibility of a cure to brain damaged areas resulting from NSC carrying out long-distance movement to the required to perform at least active or resistant movements in order for rebirth or increase of neural stem cells and plasticity of an adult central nervous system. Also, the report made by Jaime Imitola and others show the possibility of a cure to brain damaged areas resulting from NSC carrying out long-distance movement to the damaged area of a central nervous system with correct directionality.

30 to 40 years ago, many PT and OT accepted the PNF method as theoretical, yet enforced it. However, there is no fruitful outcome compared with the older popular technique. Netting a mysterious result, it seems that this question is solved by the reversible elucidation of the above adult central nervous system. Researchers were questioning the inexplicable result of the neuromuscular facilitation technique, which captivated all the PT and OT some 30-40 years ago, ending up in no distinguished effect and having almost no difference from the muscular power reinforcing method from previous times. However, there are indications that these kinds of questions are to be solved by elucidation of the plasticity of an adult central nervous system mentioned above.

Takizawa, Shigeo ${ }^{12)}$, has predicted that reproduction of the human central nerves are being generated from the effect of individual movement rehabilitation according to the program by the seating position of one operator and patients of a large number shown in Chapter III clause 3.

\section{An example considered to be successful in PNF}

The physical therapists who perform the neuromuscular facilitation technique to the affected side, propose that a program will be better by utilizing it. Therapists tend to avoid the neuromuscular facilitation technique to an unaffected side. However, the rehabilitation services which focus on not becoming bedridden or the deterioration of physical strength should be considered more than making an issue of patterns of a percentage of elderly people.

\subsection{Bilateral and Symmetrical Pattern Exercises for the training of hemiplegic upper extremity.}

Jill Whital and others ${ }^{13), 14)}$ have reported that there was a positive effect to those who had an onset of apoplexia cerebri within an average of four years by making bilateral Symmetrical Pattern Exercises perform to training of the hemiplegia upper arm. It carried out for six weeks 3 times per week once for 1 hour a day, and the influence movement has on brain function using a functional Magnetic Resonance Imaging (fMRI) was investigated. fMRI inspection was carried out before and six weeks after the treatment. A new part of the brain was activated in 6 patients $(67 \%)$ out of 9 who received training. Most of them are on the healthy side and new brain formation seems to appearing. On the other hand, researchers say, "In the group who received treatment on the affected side only, no new activated part was seen." As stated to (3) of II., Symmetrical Pattern of the unaffected contra lateral upper limbs produced the resistance movement of the 
affected upper limbs by the irradiation, and this is interpreted to have raised cell activity on the brain level. New knowledge is that the new route by rule of an unaffected side brain was developed at least here. This means that it did not stop the reversibility for spasticity, contracture, rigidity, atrophy, etc. It usually occurs in about one year but it was extended in a little more than 4 , meaning that it prevented a reversibility inhibitory factor. Although the upper limbs of the effect of irradiation from the opposite side are not as fruitful as the lower limbs, in the reciprocal (alternative) or symmetrical pattern, it is not always the case.

As described before, when getting the irradiation outcome to the affected distal muscle, the one from the proximal synergist of the contra lateral unaffected muscle is usually much stronger than that from the affected isonomic muscle. It can make the affected distal muscles actively contract, avoiding the disuse atrophy contracture and the antagonists spasticity. That can be used to train the affected distal muscle to be able to contract against its antagonist, attaining the reciprocal motion and preventing the disuse atrophy, contracture and the antagonists' spastcity.

\subsection{Movement apparatus performing by repeating extension joint movement of both the upper limbs.}

Namba ${ }^{15)}$ developed the movement apparatus which repeats extension joint movement of the both upper limbs for the training of the upper limbs of hemiplegia. A subject is made to grasp the grip with both hands, and perform push-pull exercises. Improvement was found by controlling the upper limbs and the voluntary action of fingers at an early stage with the spasticity of the affected side upper limbs being reduced. It is said that a family can use it since it is simple repetitive exercises, and it also tends to be used in an institution since this does not require assistance.

\subsection{Walk re-acquisition of an aged disabled person}

According to the rehabilitation of an aged disabled person, described below is an example of walk re-acquisition aged disabled persons, after lapsing into disuse and becoming bedridden. This rehabilitation was done by one therapist to a large number of patients, by using a device in sitting position.

(i) Bilateral and Symmetrical Pattern Exercises for the training of hemiplegic lower extremity. A physical therapist, Takizawa invented a simple device many years ago, which moves a leg joint and an articulation genus at the initiative of the unaffected side in a sitting position. Takizawa insists that by using this device with perseverance, good ambulatory ability is possible to gain, in addition to the control of the leg joint clonus. There were few people interested in these devices ${ }^{16)}$ because it was too simple.

\section{(ii) Systemic ADL exercises including programmed upper extremities training}

It is also considered to be one of the reasons which caused more effect on the whole in order to prevent the common movements for the elderly people such as (a) Poor childish operation, and (b) The operations for the old people who are unstable and apt to fall down, the method of systemic ADL exercises ${ }^{17)}$ and the fruitful quality of life were added.

\section{(iii) Evaluation research}

The following effect is asserted as a result of Bilateral and Symmetrical Pattern Exercises for the training of hemiplegic lower extremity of above-mentioned (i), upper-limbs mutual repetitive-exercises training by the above-mentioned (ii) upper-limbs training device, and systemic ADL gymnastics, those which Takizawa carries out. - Takizawa has insisted on few appearances of spasticity. (Musculus quadriceps femoris, musculus triceps surae, especially the latter). ${ }^{18)}$

- There were many examples which had a result of patients being able to walk even if the period was over 6 months. ${ }^{19)}$

- It could be used also for the elderly people aged 80 and over, and was effective. ${ }^{20), 21)}$

The report of this method is performed by Takizawa, Shigeo and others. ${ }^{22), 23), 24)}$ Moreover, research by public funds was done in Health Care Facilities for the Elderly Requiring Long-term Care (HCFE), "Shonan no oka" where the author was working as a representative. In the research, Morita ${ }^{25)}$ performed qualitative evaluation, Ushizawa ${ }^{26)}$ and others conducted statistical analysis (multiple-comparison method: t-test, Sign-test), and remarkable validity is accepted. The patients mentioned above, who were in Shonan-No-Oka HCFE and were rehabilitated by the programmed rehabilitation, have done a statistical evaluation on effects in order to find the pattern of rehabilitation effects.

These researches ${ }^{25), 26)}$ that make Kimura a representative were done to a total of 51 persons for total of 4 times from June of 2000 to February, 2001. The period when rehabilitation was offered was uneven. Many of evaluation candidates had merged the motor palsy or the slight dementia resulting from cerebrovascular disorder. 44 examples which the rehabilitation effect can be measured were made 
applicable to analysis in the evaluation period. From the $\mathrm{FIM}^{* * *}$ synthesis score, although the rehabilitation effect was not detected in either case, finding for "a walk and a wheelchair" was judged effective by three kinds of methods including t-test when examined topically. A conclusion about "defecation", "toilet operation", and "understanding" were also approved of. While the results of using the total of all the items of FIM points by chart as mentioned above, 'L. walking, wheelchair' was evaluated as significantly effective in either assay, and although the effect of ' $\mathrm{H}$. Bowel management' was admitted in Sign approval due to examination of items respectively ${ }^{26)}$. Moreover, Makita ${ }^{27)}$ carried out the examination for the randomized control test in the HCFE, and has accepted and reported its validity.

\section{The advantage and mechanism of PNF led by unaffected side}

Like previous statements, methods with an effect are either bilateral or alternans repetitive exercise. It admits the possibility of assisting muscular power reinforcement, maintaining a similar level without that the theory of Successive Induction ${ }^{*}$ can be used from an early stage maintaining the balance of agonists and antagonists without accelerating mutual spasticity. It is presumed also from the appearance of spasticity several weeks after the appearance of paralysis. It is strengthened much more, and this will be repeated by turns of successive induction of contraction of the antagonist that precedes work of agonist muscle, and agonist muscle will be gradually strengthened. The center of a rebirth of an unaffected side brain will also be established.

In consideration of above topics, (Fig.2),

(1) PNF of an unaffected side is added to the affected side PNF, and strengthens contraction of a peripheral muscle. More strong facilitation is obtained than in the case led by an affected side.

(2) Since Successive Induction is performed simultaneously, strengthening of an antagonist is also simultaneously possible, since contracture, rigidity, spasticity, etc. can be prevented, and there is an advantage which can be used also for the old over a long period of time.

(3) It is proved by the animal level that a new center is produced not only in an affected side but in an unaffected side of the brain. Successive and alternative exercises control the appearance of spasticity and are considered to urge favorable recovery.

(4) Although it is unknown whether mobilization of a neural stem cell is made easy, the activity period is thought to be extensible for a long time, and a higher goal than achieved is expected.

(1) Agonist

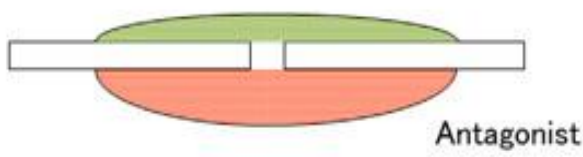

(2) Atrophy and deformation on the agonist

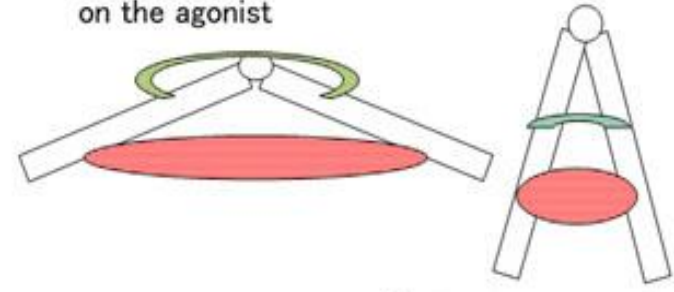

(3) Displacement of the agonist

Fig 1, an agonist muscle and an antagonist

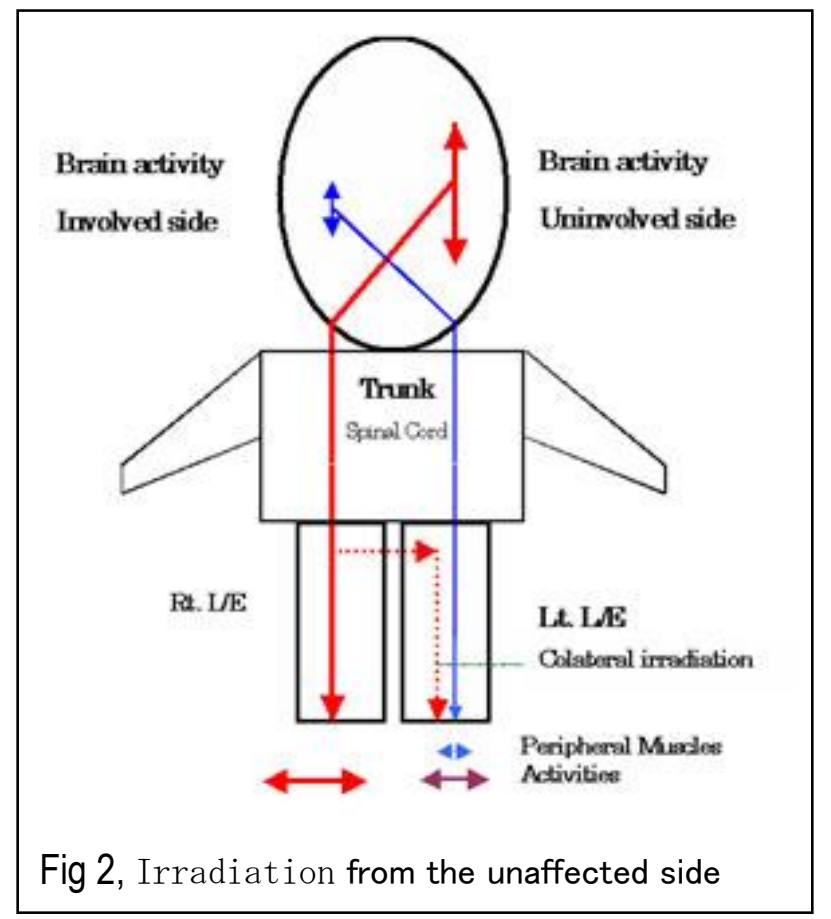




\section{Conclusion}

PNF used regularly and conventionally is an outstanding theory. However, it is considered that there is not enough reward for the efforts with the conventional method. The paralyzed muscles do not recover muscular power in passive exercise, there cannot be recovery, and if possible the active resistive exercise at least is necessary for the recovery of paralyzed muscles. In movement led by the paralysis side, when paralysis is severe, the peripheral paralysis muscles do not reach to the level of effective active movement, but result in atrophy and disuse, and might end in the result that increases the spasticity of an antagonist.

However;

(i). If it's carried out by unaffected side initiative in response to the influence of Colateral irradiation from the unaffected side, the contraction of the affected side peripheral muscles become stronger.

(ii). It can build a new compensatory center in the unaffected side brain.

(iii). Agonist muscle and an antagonist can be balanced by the ability of spasticity to be further controlled by making reciprocal pattern do, and it is thought that the example which can lengthen the time of plateau to several years.

Furthermore, although promoting NSC to the damaged area by this procedure is unknown, it is possible to delay the coming of the plateau level by reducing atrophy and disuse of paralytic limb, and to extend a recoverable period.

\section{Reference}

1) Yukito Shinohara, et al : 5 society joint apoplexy medical treatment guideline (the provisional version). 2003. http://www.neurology-jp.org/guideline2003/5_0201.html.

2) Dickstein R, Hocherman S, Pillar T, Shaham R. Stroke rehabilitation: three exercise therapy approaches. Phys Ther 1986; 66: $1233-1238$.

3) Logigian MK, Samuels MA, Falconer J, Zagar R: Clinical exercise trial for stroke patients. Arch Phys Med Rehabil 1983; 64: 364-367.

4) Stern PH, McDowell F, Miller JM, Robinson M. Effects of facilitation exercise techniques in stroke rehabilitation. Arch Phys Med Rehabil 1970; 51: 526-531.

5) Wagenaar RC, Meijer OG, van Wieringen PC, Kuik DJ, Hazenberg GJ, Lindeboom J, et al. The functional recovery of stroke: a comparison between neuro-developmental treatment and the Brunnstrom method. Scand J Rehabil Med 1990; 22: $1-8$.

6) Goolsby J, Marty MC, Heletz D, Chiappelli J, Tashko G, Yarnell D, Fishman PS, Dhib-Jalbut S, Bever CT Jr, Pessac B, Trisler D.: Hematopoietic progenitors express neural genes. Proc Natl Acad Sci U S A. 2003 Dec 9;100(25):14926-31. Epub 2003 Nov 21.

7) Van Praag H, Kempermann G, Gage FH: Running increases cell proliferation and neurogenesis in the adult mouse dentate gyrus. Nat Neurosci. 1999 Mar;2(3):266-70.

8) Kempermann G, Kuhn HG, Gage FH: More hippocampal neurons in adult mice living in an enriched environment. Nature. 1997; Apr 3;386(6624):493-5.

9) Chemokine secreted by inflammation neuron attract transplant nerve stem cells. Medical Tribune, February 17, 2005 PP1, Medical Tribune. Inc.(JP)

10) Imitola J, Snyder EY and Khoury SJ: Genetic programs and responses of neural stem/progenitor cells during demyelination potential insights into repair mechanisms in multiple sclerosis. Physiolog.Genomics 2004; 14:171-197.

11) Imitola J, Raddassi K, Park KI, Mueller FJ, Nieto M, Teng YD, Frenkel D, Li J, Sidman RL, Walsh CA, Snyder EY, Khoury SJ. Directed migration of neural stem cells to sites of CNS injury by the stromal cell-derived factor 1 -alpha/CXC chemokine receptor 4 pathway. Proc Natl Acad Sci U S A .2004; 101:18117-22.

12) Takizawa, Shigeo: AN APPLICATION OF THE CIVIL TECHNOLOGY TO THE SUSTAINABLE AGED SOCIETY. Biophilia Rehabilitation Journal 2004; 2-1: pp1-24 Reprint as Review, Application of the Social Technology to the Sustainable Aged Society, BIOPHILIA 2015-1, PP60-80

13) Nerve pathways are activated with the repetitive exercises of both arms. Medical Tribune. Inc. Medical Tribune, January 13, 2005 PP1. (JP)

14) Jill Whitall, PhD; Sandy McCombe Waller, MS, PT, NCS; Kenneth H. C. Silver, MD; Richard F. Macko, MD: Repetitive Bilateral Arm Training With Rhythmic Auditory Cueing Improves Motor Function in Chronic Hemiparetic Stroke. Stroke 2000;31:2390.

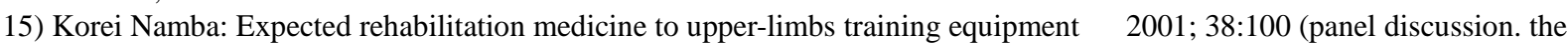
38th The Japanese Association of Rehabilitation Medicine scientific meeting)

16) Kyoko Takizawa : Use report of a leg joint leg training implement. 10th Rehabilitation Engineering Conference Proceedings 1995; pp.119-122

17) Kyoko Takizawa : Care prevention and rehabilitation. 8th Biophilia Rehabilitation Conference Proceedings (keynote lecture IV) 2004;pp.8

18) Kyoko Takizawa: Actual condition of TAKIZAWA method Rehabilitation in the acute term hospital -- expecting a new challenge --. 9th Biophilia Rehabilitation Conference Proceedings (keynote lecture II ) 2005; pp.4.

19) Kijima H, Kanai S, Takizawa K, Takizawa S, et al. The Rehabilitation Trial for the acquisition of walking form bedridden 
at the bedridden elderly hospital. Journal of the Japanese Clinical Orthopedic Association. Vol. 23. NO. 2 JUNE. 1998: 186-191.

20) Takizawa, S, Kimura. T, Kijima, H, Takizawa K, et al:Ambulation from bedridden (The case report of a bedridden inpatient of double-hemiplegia).ISPRM. (1) 2001; Ferrarese, Monduzzi Editore, pp747-757: Reprint as Case report, Ambulation from Bedridden - Patient with Double Hemiplegia, BIOPHILIA 2015-1, PP16-18.

21) Takizawa, S. (Editor and author) Acquisition of walking from bedridden. Book published. Civil Publishing Inc. Fujisawa. 1996.

22) Takizawa, Shigeo, Tetsuhiko Kimura, Hideo Kijima, Yuzou Okamoto, Kentaro Nagaoka, Kyoko Takizawa, The development of devices for the MOTIVATIVE exercise of impaired extremities, CSUN's sixteenth Annual International Conference, Los Angeles, 2000. http://www.csun.edu/cod/conf/2000/proceedings/csun00.htm: Reprint as Research, BIOPHILIA 2015-1, PP7-11.

23) Takizawa, S, Kimura, T, Kijima, H, Okamoto, Y, Nagaoka, K, Morita, Y, Endo, S, Nagasawa, H, Makita, M, Takizawa, K, Biophilia Rehabilitation and Proposition of the solution to the aging crisis, ISPRM. (1), Ferrarese, Monduzzi Editore, 729-736, 2001: Reprint as Research, Re-acquirement of Walking from Bedridden by the Motivative Exercise and the Takizawa Method and Proposition of the Solution to the Aging Crisis, BIOPHILIA 2015-1, PP12-15.

24) Takizawa, S, Keeping the Elderly in Motion, Reha. Management international, Los Angels, Medical World Communications, 48, 2000.

25) Yoshiko Morita. Survey research for the ADL improvement and independence of aged patient of bone fracture of the lower extremities. (Editor Kimura T),Book published. Civil Publishing Inc. Fujisawa. 2001;pp36.

26) Kenji Ushizawa $\cdot$ Shigeo Takizawa $\cdot$ Hiroshi Nagasawa Mitsuyo Makita $\cdot$ Tetsuhiko Kimura $\cdot$ Kyoko Takizawa, Statistical Evaluation of Rehabilitation to the Disabled Elderly based Takizawa-Program, Biophilia Rehabilitation Journal, 2-1, p71-80, 2004.

27) Mitsuyo Makita, An Examination by Randomized Control Test of the effectiveness of exercise therapy (Takizawa Program) on bedridden elderly patients(2), Proceedings of the Fourth International Conference of the Biophilia Rehabilitation Academy, pp21, 2005.

\{Notes\}

*Niche: Very small environment for the cultivation of NSC (Micro environment)

${ }^{* *}$ Successive induction: If the maximum contraction is carried out for an antagonist just before a line of operation works, and the agonist is fully stretched, it will become more powerful than usual.

${ }^{* * * *}$ FIM: the Functional Independence Measure introduced to Japan by Keio University

Corresponding author:

Shigeo Takizawa

(E-mail: takizawa@ biophilia.biz)

Biophilia Institute

4-24-5 Shonandai Fujisawa-shi, Kanagawa Pref. 252-0804

Received: December 1, 2005, Reviewed: March 1, 2006, Published: March 31, 2006

This article was published as above in the Japanese Journal as an English original article. The Japanese one became a predecessor journal of the BIOPHILIA. As a memorial edition for the getting ISSN-L and for a help of the English readers to find this article, we permitted to reprint and publish in the BOPHILIA journal.

The permission date by accomplished the review on February 17, 2015.

The Biophilia editorial board obtained the publication request and copyright holder's permission, and published this article after the review. 